\title{
SIMD-Matcher: A SIMD-based Arbitrary Matching Framework
}

\author{
PING WANG, FEI WEN, PAUL V. GRATZ, and ALEX SPRINTSON, Department of Electrical \\ and Computer Engineering Texas A\&M University, USA
}

\begin{abstract}
Packet classification methods rely upon matching packet content/header against pre-defined rules, which are generated by network applications and their configurations. With the rapid development of network technology and the fast-growing network applications, users seek more enhanced, secure, and diverse network services. Hence it becomes critical to improve the performance of arbitrary matching operations.

This article presents SIMD-Matcher, an efficient Single Instruction Multiple Data (SIMD) and cachefriendly arbitrary matching framework. To further improve the arbitrary matching performance, SIMDMatcher adopts a trie node with a fixed high fanout and a varying span for each node depending on the data distribution. The trie node layout leverages cache and modern processor features such as SIMD instructions. To support arbitrary matching, we first interpret arbitrary rules into three fields: value, mask, and priority. Second, to support insertion of randomly positioned wildcards to arbitrary rules, we propose the SIMD-Matcher extraction algorithm to process the wildcard bits. Third, we add an array of wildcard entries to the leaf entries, which store the wildcard rules and guarantee the correctness of matching results. Experiments show that SIMD-Matcher outperforms GenMatcher under large-scale ruleset and key set, in terms of search time, insert time, and memory cost. Specifically with 5M rules, our method achieves a 2.7X speedup on search time, and the insertion time takes $\sim 7.3$ seconds, gaining a $1.38 \mathrm{X}$ speedup; meanwhile, the memory cost reduction is up to $6.17 \mathrm{X}$.
\end{abstract}

CCS Concepts: • Networks $\rightarrow$ Network architectures; Network algorithms; Packet classification; Network simulations; Network performance analysis; Packet-switching networks;

Additional Key Words and Phrases: Arbitrary matching, SIMD optimization, performance trade-off

\section{ACM Reference format:}

Ping Wang, Fei Wen, Paul V. Gratz, and Alex Sprintson. 2022. SIMD-Matcher: A SIMD-based Arbitrary Matching Framework. ACM Trans. Arch. Code Optim. 19, 3, Article 30 (May 2022), 20 pages.

https://doi.org/10.1145/3514246

\section{INTRODUCTION}

Packet classification is the critical component in a switch network, which classifies/forwards internet packets to flows according to the pre-defined rules generated by the different network applications and their configurations [10]. With the advent of SDN technology, the applications have

Authors' address: P. Wang, F. Wen, P. V. Gratz, and A. Sprintson, Department of Electrical and Computer Engineering, Texas A\&M University, 400 Bizzell St., College Station, TX, 77840; emails: freesummer@tamu.edu, fei8wen@gmail.com, pgratz@gratz1.com, spalex@tamu.edu.

Permission to make digital or hard copies of all or part of this work for personal or classroom use is granted without fee provided that copies are not made or distributed for profit or commercial advantage and that copies bear this notice and the full citation on the first page. Copyrights for components of this work owned by others than the author(s) must be honored. Abstracting with credit is permitted. To copy otherwise, or republish, to post on servers or to redistribute to lists, requires prior specific permission and/or a fee. Request permissions from permissions@acm.org.

(C) 2022 Copyright held by the owner/author(s). Publication rights licensed to ACM.

1544-3566/2022/05-ART30 \$15.00

https://doi.org/10.1145/3514246 
become more flexible since the control plane is decoupled from the data plane. This separation increases the demand for software-based generic packet classification methods. In traditional switch networks, rules consist of five fields: source IP address, destination IP address, source port number, destination port number, and protocol [2]. However, in an SDN switch network, the rules have more fields as defined in OpenFlow [11], a de facto standard of SDN. OpenFlow defines the communication mechanism and message format between the control plane and the data plane. The rules in OpenFlow have 15 required fields and dozens of optional fields. The latest OpenFlow ver. 1.5 defines 45 different flow match fields, in which about half of them involve wildcards [30]. Therefore, the multi-field packet classification problem becomes more complex and challenging [14, 26, 29].

Packet classification is done by matching packet header fields in computer networks. Apart from header field matching, packet classification can also be implemented by content matching that involves wildcards, in other domains such as bioinformatics [25], information retrieval [4], dictionary query [6], and so forth. However, these content arbitrary matching applications are outside the scope of this article.

While there exists plenty of research on packet matching, most of this work focuses on prefix and range matching [28]. With the prevalence of High-Performance Computing (HPC) applications, the software-defined network technique will be deployed in HPC networks [17], requiring high-performance packet classification [8]. Field matching schemes allow wildcards for the fields that are not associated with particular matches. Different applications generate various multi-fields, along with wildcard rules that come with diverse distribution of the wildcard bits. For example, if matching is only based on MAC address or UDP/TCP port, other irrelevant fields such as IP addresses are wildcarded [13]. Furthermore, in OpenFlow-based data center networks, a proactive routing application utilizes wildcard rules to balance the traffic flows between switch ports [18]. The diversity of wildcard matching applications renders multi-field packet classification an essential challenge for SDN devices. There are four different matching types for packet classification: exact match, prefix match, range match, and arbitrary match [32]. Prefix match and arbitrary match both contain wildcard bits. However, the wildcard bits of prefix match are located at the end of a rule, whereas arbitrary match may have wildcard bits at any bit position. Since all matching types can be represented in an arbitrary match, the essential task is to improve the generic arbitrary matching performance.

There are few prior works on arbitrary matching. Meiners et al. proposed Bitweaving [23], a hardware-based compression approach to reduce the number of ternary content-addressable memory (TCAM) rules. Bitweaving utilizes the TCAM, which is expensive, power hungry, and capacity limited. Therefore, the Bitweaving method is not suitable for large data applications. We introduced GenMatcher [32], a software-based generic clustering-based arbitrary matching framework, which is implemented with a binary trie data structure. Compared to the linear search and bitweaving [23] method, GenMatcher gained a significant speedup on search performance. Although GenMatcher improved the arbitrary matching performance, it does not leverage the modern processor features, such as Single Instruction Multiple Data (SIMD) instructions (e.g., SSE [31], AVX [1], etc.). Further, GenMatcher has poor scalability toward the increasing size of input data: the searching time complexity grows linearly along with key length, while the binary tire memory consumption grows exponentially. Ultimately, with the demand for high-performance matching from big data and HPC applications, GenMatcher restricts the room for improvement in search performance and memory cost. Thus, the high-performance arbitrary matching is still an open problem.

Binna et al. proposed the height-optimized trie (HOT) [5] data structure, which takes advantage of SIMD instructions and modern cache features that exploit data localities to accelerate the searches and reduce memory usage. Unfortunately, HOT cannot be directly applied for arbitrary 
matching because HOT does not support wildcard rules. In addition, HOT is incapable of prioritizing results. Since wildcard matching can have multiple matching results, the priority feature is needed to choose the correct candidate, usually with the highest priority. Thus, there are no prior works we are aware of that leverage modern processor features supporting arbitrary matching. To fill this gap, we aim to achieve a high-performance search throughput and memory cost by utilizing the modern processor features for arbitrary matching.

A key to achieving high matching performance is to ensure that the matching data structure can utilize the modern CPUs' advanced features. This article proposes SIMD-Matcher, a generic SIMD-based arbitrary matching framework that utilizes modern processor features and SIMD instructions to improve the matching performance in search time and memory cost. Our proposed SIMD-Matcher extends the HOT data structure presented by Binna et al. [5], in which the trie node is composed of a fixed upper bound of a binary trie node. The trie node has a data-dependent span and a fixed maximum fanout, enabling a consistently high fanout for arbitrary key distributions and efficient search using the SIMD feature.

To process arbitrary matching, we propose a novel mechanism to deal with the wildcard rules. First, we defined wildcard rule representation [32]. After the interpretation of wildcard rules, we can insert them into the HOT data structure. Second, HOT only carries partial bits of a rule, represented by the discriminative bits, which efficiently reduces the memory cost. Since the wildcard rules have $*$, which can be either 1 or 0 , we propose a novel algorithm to extract the effective partial bits based on the rules' distribution. Third, to guarantee the integrity of wildcard rules' information, we must store the rules' complete bits in the corresponding leaf nodes.

Wildcard rule representation: We design a rule representation method that can interpret wildcard rules into three fields. This representation allows us to insert the wildcard rules into the trie data structure.

Challenges: HOT as originally conceived was able to represent a rule with only partial bits, which reduces the memory footprint. However, HOT cannot be directly applied to arbitrary rules that have wildcard bits, because the partial bits derived from the difference among all rules' determined bits, while a wildcard bit can be randomly translated to either 0 or 1 . To enable the wildcard matching operation, we design a SIMD-Matcher extraction algorithm that can obtain a wildcard rule's partial bits to guarantee the matching outcome. As wildcard rules can cause multiple matching rules for an incoming key, we need to choose the highest priority to get the correct matching result. Therefore, we need to use some extra space for the leaf node to store the wildcard rules. Our goal is to utilize the SIMD feature to improve the performance for efficient arbitrary matching. To implement the efficient arbitrary matching, we must address several challenging technical problems:

- We must modify the HOT data structure to support wildcard matching.

- We must develop a partial bits extraction algorithm to choose the affected discriminative bits from the corresponding node's discriminative bits set.

- We must guarantee that the arbitrary matching result is correct; i.e., the candidate with highest priority will be chosen when there exist multiple matching candidates.

Contributions: SIMD-Matcher is an advanced generic arbitrary matching approach that can take advantage of modern processors' features. Our SIMD-Matcher has the following features:

- We introduce SIMD-Matcher, a generic SIMD-based arbitrary matching framework, which can process any form of matching used in packet classification utilizing the SIMD feature.

- We develop a novel SIMD-Matcher extraction algorithm to obtain the effective partial bits of a wildcard rule. 
- Experiments show that SIMD-Matcher achieves search time speedup by utilizing the SIMD feature on average by $2.7 \mathrm{X}$ compared to GenMatcher, and up to 6.17X reduction for the memory footprint.

\section{BACKGROUND}

Arbitrary matching can be implemented either by hardware-based or software-based approaches. TCAM [20] is a hardware-based solution that can process the arbitrary rules in parallel. Nonetheless, TCAM is power hungry and expensive, and it cannot scale with the number of rules [33], especially for the big data and HPC applications. This article will focus on the software-based approach. The goal is to develop a data structure that is efficient for high-performance arbitrary matching.

We have witnessed the rapid evolution of processor architectures and memory systems, whose new features have been proven beneficial to arbitrary matching [3, 16, 19, 21]. Meanwhile, trie is a lexicographic data structure where nodes can share their common prefix. Even with the increasing size of datasets, the trie height is independent of the rule size; instead, it is determined by the length of the rule and the node design. In particular, traditional tries cannot be directly used in arbitrary matching where wildcards may appear at any bit positions. However, the trie can be applied for the arbitrary rules via certain transformations. Our previous work proposed a generic arbitrary matching framework, GenMatcher. GenMatcher can convert the arbitrary rules into prefix rules inserted into a binary trie data structure. The search performance of a binary trie depends on the trie depth, which equals the length of a rule. Hence, we need to decrease the trie height in order to improve the search performance. In this article, we will concentrate on optimizing the trie data structure to achieve high-performance arbitrary matching.

\subsection{The Benefits of SIMD for Matching}

Modern processors feature wide vector units, which can be leveraged through SIMD instructions [15], exploiting data-level parallelism. Intel's Streaming SIMD Extensions [31] expand the register width from 32 bits to 128 bits to support processing four single-precision floating-point numbers at one time. The subsequent Intel Advanced Vector Extensions (AVX) [1] and Intel AVX2 further extended to process 256 bits of data at one time. The successor Intel Advanced Vector Extension 512 is able to operate on 512 bits of data with a single instruction.

SIMD instructions support various operations. This article will mainly use comparison operations, bit operations, and register I/O operations. In contrast to comparing operations of scalar types, there cannot be a single true or false result for a SIMD comparison. Instead, there will be multiple Boolean values in the result. The register width determines the size of this vector of Boolean values. A vector of Boolean values is named a mask. For bit operations, we utilize Boolean logic operations, selective bit moving operations, bit counting, and bit extract operations. Concerning the register I/O operations, we utilize broadcast load operations.

In this article, we employ SIMD instructions to improve the performance in search performance and memory cost.

\subsection{Relationship with Prior Art}

Table 1 shows the complexity comparisons between all the following trie data structures, where $W$ is the key length, $M$ is the span size, and $T^{1}$ is the trie node size. The space complexity in Table 1 is based on the worst case.

$\overline{{ }^{1} \text { The trie node }}$ size of each trie data structure in Table 1 is unique, determined by its trie node layout. 
Table 1. Complexity Comparisons of the Different Trie Data Structures

\begin{tabular}{|c|c|c|c|}
\hline & Trie Height & Space Complexity & SIMD Feature \\
\hline Binary trie & $O(W)$ & $O\left(2^{W} \cdot T\right)^{2}$ & No \\
\hline Patricia trie & $O\left(W^{\prime}\right)^{3}$ & $O\left(2^{W^{\prime}} \cdot T\right)$ & No \\
\hline$M$-ary trie & $O(W / M)$ & $O\left(\left(2^{M}\right)^{(W / M)} \cdot T\right)$ & Yes \\
\hline ART trie & $O\left(W / M^{\prime}\right)^{4}$ & $O\left(\left(2^{M^{\prime}}\right)^{\left(W / M^{\prime}\right)} \cdot T\right)$ & Yes \\
\hline HOT trie & $O\left(W^{\prime \prime}\right)^{5}$ & $O\left(2^{W^{\prime \prime}} \cdot T\right)$ & Yes \\
\hline
\end{tabular}

2.2.1 Binary Trie. GenMatcher [32] groups rules and converts wildcard rules into prefix rules, which are later inserted into a binary trie data structure. For a 64-bit integer ruleset, the worst trie height will be 64 if there are no wildcard rules. During the search process, we have to search each bit sequentially to get a matching result. The trie heights should be reduced for optimized performance. Therefore, we need to optimize the trie data structure from cache efficiency and modern processor utilization to reduce the height.

2.2.2 Patricia Trie. We explore the Patricia trie (or radix tree) [24], a compressed version of a trie. In a binary trie, for a 64-bit integer, each trie node can have up to two edges: bit- 0 and bit-1. Each non-wildcard bit will be stored in the trie as a trie edge, while in a Patricia trie, multiple bits can be stored in the Patricia trie as a single edge. Thus, multiple binary nodes can be compressed into one node, which reduces the trie height and decreases the number of nodes, resulting in saving memory.

2.2.3 M-ary Trie. To further decrease the trie height, we study the $M$-ary trie, which expands the span to $M$ bits in contrast to the 1-bit span of the binary trie. The maximum number of children of each trie node is $2^{M}$. Modern CPUs can execute multiple comparisons with a single SIMD instruction, which expedites the $M$-ary search. Consequently, the number of cache misses is reduced because $2^{M}$ comparisons can be performed for each cache line loaded from main memory [19]. Hence, compared with the binary trie, the $M$-ary trie is not only more effective in searching by utilizing SIMD instruction but also cache-friendly. Furthermore, the trie height is reduced from $W$ to $W / M$, where $W$ is the key length. However, as the span size increases, the memory cost increases exponentially because each node is allocated $2^{M}$ pointers in an array. Recently, some big data applications are more sparsely distributed, which causes a large amount of memory to be wasted. Thus, for different applications, we need to adopt varying span sizes to save memory costs. Therefore, we explore the adaptive radix tree (ART) [19] data structure next.

2.2.4 ART Trie. While a traditional radix tree requires the tradeoff of tree height with memory cost by setting a globally valid span parameter, ART proposes a node represented with a varying span size. The number of child nodes determines the size. If a node has many child nodes, ART assigns an enormous span to this node; otherwise, it obtains a smaller span. Thus, the adaptive nodes lead to a lower memory cost by decreasing the number of empty pointers. However, since the trie height is determined by the key length and the span size, the ART trie might be unbalanced due to the various span sizes.

\footnotetext{
${ }^{2}$ Here the binary trie is a complete tree and each trie node has two pointers.

${ }^{3}$ The Patricia trie is a path-compressed trie whose height might be reduced based on the data distribution. On average, $W^{\prime} \leq W$.

${ }^{4}$ Since the ART trie node can have different span sizes, $M^{\prime}$ represents its average span size.

${ }^{5} W^{\prime \prime}$ is determined by the data distribution, and $W^{\prime \prime}<W^{\prime}$.
} 
For all the tries mentioned earlier, the node and span bits ${ }^{6}$ are fixed elements. If the dataset is sparsely distributed, the data structure is likely an unbalanced trie. To solve this problem, we need to explore a radix tree with nodes of equal fanout and various span bits. Meanwhile, with the increasing speed gap between cache access and main memory access, it's a crucial task to exploit memory reference localities to improve cache behaviors and overall performance of in-memory data processing [27]. For a binary trie, trie search presents significant challenges due to irregular and unpredictable data accesses during trie traversal [12]. The typical size of a block/cache line is 64 bytes [9]. The processor will read or write an entire cache line when any location in the 64-byte region is read or written [9]. Good memory reference locality leads to fewer cache misses, which can reduce the overall memory access time.

2.2.5 HOT Trie. To leverage the memory cost and search performance, Binna et al. [5] propose a HOT data structure, which retains a consistently high fanout and reduces the overall height. HOT is a new data structure designed for in-memory databases, with the purpose of achieving high performance and low memory cost. A conventional trie node has fixed span bits and datadependent fanout, while the HOT trie node features data-dependent span bits and a fixed maximum fanout $^{7} k$ [5]. The design of $k$ is set to 32 since 32 is an optimal value considering the tradeoff between the cache locality ${ }^{8}$ and fast update. Thus, each trie node has the same maximum number of children, but each child may cover different bit positions.

HOT saves the memory cost for storing keys by introducing discriminative bits. The discriminative bit is defined as the bit position that has different values. For the integer dataset, the values are " 0 " and " 1 ." If any bit position has both values 0 and 1, this bit position will be included in the discriminative bits set. The partial key is extracted from the whole key using these discriminative bits. For the node layout, HOT utilizes three different sizes of the partial key: 8-bit, 16-bit, and 32-bit. The data distribution determines the node type's selection since the size is equal to the number of discriminative bits among all the keys/rules in the dataset. For example, comparing bit string "10" with "11," the discriminative bit is bit-0 position (least significant bit). If here comes another bit string " 00 ," we obtain another new discriminative bit position, bit-1. If the dataset only has these three rules, the 8-bit format of the node is selected since we only have two discriminative bits: bit- 0 and bit- 1 . The set of discriminative bits is anticipated to grow along with the dataset size, requiring a bigger node size.

The details of insert and search operation are explained in Section 3. First, HOT defines the extract operation to obtain all the discriminative bits from the input dataset. Next, HOT compares the extracted partial keys/rules to the inserted partial data. The comparison operations occur in both insert and search operations. The HOT node has a fixed maximum of 32 children, which is efficient for utilizing SIMD instructions. It can compare one search key with $32 \times 8$-bit keys in parallel by utilizing AVX. HOT searches through the current data structure during the insert process to check if the rule is already inserted. If it is true, the insert operation is finished and returns 0 . Otherwise, the procedure will find the mismatched BiNode (binary trie node) and create a new discriminative bit using this mismatched bit position. Therefore, each inserted rule has a unique combination of discriminative bits, demonstrating a customized span's property. HOT extracts discriminative bits from the search key during the search process and then traverses through the HOT to search for any mismatch. The optimization for cache and SIMD efficiency leads to high search efficiency,

\footnotetext{
${ }^{6}$ Span bits denote the specified bit positions that the trie node edge crosses.

${ }^{7}$ In any tree data structure, the fanout of a node is defined to be the number of children the node has. The fanout of a tree is defined to be the maximum fanout of any node in the tree.

${ }^{8}$ E.g., given that each data entry has 16 bits and the cache line size is 64 bytes, if we load a $32 \times 16$-bit type of data to the cache, there is only one miss for the first entry. The remaining 31 entries will be all cache hits.
} 
Table 2. An Example of a Wildcard Ruleset

\begin{tabular}{|c|c|c|c|}
\hline Rules & Value & Mask & Priority \\
\hline R1: 00101 & 00101 & 00000 & 1 \\
\hline R2: 10110 & 10110 & 00000 & 2 \\
\hline R3: 11110 & 11110 & 00000 & 3 \\
\hline R4: 11101 & 11101 & 00000 & 4 \\
\hline R5: $0^{*} 11^{*}$ & 00110 & 01001 & 5 \\
\hline R6: $1^{*} 10^{*}$ & 10100 & 01001 & 6 \\
\hline R7: $0^{*} 0^{* *}$ & 00000 & 01011 & 7 \\
\hline R8: $0^{*} 1^{* *}$ & 00100 & 01011 & 8 \\
\hline R9: $1^{* * * *}$ & 10000 & 01111 & 9 \\
\hline
\end{tabular}

which renders the HOT data structure as an excellent framework for our wildcard matching. This article proposes SIMD-Matcher, which extends the HOT data structure to support arbitrary matching operations.

A rule may have wildcards for an arbitrary matching operation, e.g., $101^{* *} 0$, where the wildcards * are not located at the end of the bit string. HOT only inserts the discriminative bits that are necessary to distinguish the new rule from the existing rules. If a rule is an arbitrary rule, the discriminative bit sets cannot be updated correctly due to the wildcard's uncertainty. To solve this problem, we propose the SIMD-Matcher extraction algorithm to exclude the wildcard bit positions from the node's discriminative bits set. By adding this new feature, we can deal with arbitrary rules. Since we ignore the wildcard bit positions, we must store the wildcard rules in the leaf node to guarantee the correct matching. After these data structure modifications, the arbitrary rules can be inserted with their complete information. In addition, the search key can search the trie for precise matching.

\section{SIMD-MATCHER DESIGN}

In this section, we present our SIMD-Matcher design for supporting arbitrary matching. Our implementation of SIMD-Matcher is publicly available at https://github.com/freesummer/SIMDMatcher.

\subsection{Rule Representation}

We divide rules into three fields for a wildcard rule: value, mask, and priority field.

- Rule.value: The value field represents the non-wildcarded component, generated by clearing all $* \mathrm{~s}$ (wildcards) in the rule and keeping all other bits unchanged.

- Rule.mask: The mask field represents the wildcarded component, generated by parsing all *s and replacing them with 1 s, clearing all other bits.

- Rule.priority: Where there are multiple matches, we choose the highest-priority rule ( 1 being the highest). The priority is provided by the user-defined input or inferred by the order of rules initially given.

An example of a wildcard ruleset is shown in Table 2 . In the table, we note that wildcard $*$ bit is interpreted to bit- 0 in the value field and interpreted to bit- 1 in the mask field. In this article, we define the least significant bit (LSB) position as bit-0 position. The most significant bit (MSB) of rules in Table 2 is bit- 4 . Thus, the discriminative bits set of each node can have at most five bit positions. We will present the updated insertion and search operations in the following sections. 


\subsection{Insert Operation}

In order to support arbitrary matching, we design a separate procedure for insertion, which refers to extractMask, extractMaskFromSuccesiveByte, and ExecuteForDiffingKeys functions. Since a rule might have wildcard bits $*$, we need to ignore this wildcard bit position in a node to guarantee that all the rules' partial information inserted into the trie data structure is determinate. Therefore, we define a new function extractMask to extract the affected discriminative bits from the corresponding node's discriminative bit set. In this article, we denote the discriminative bits as DBbits, which are the bit positions that have a distinctive value. In the extractMask function, first, we check if the mask field is 0 ; if it is true, we return extractMask $=0$, and otherwise, we omit the wildcard bits shown in the mask field from the bit positions of DBbits.

For example, if mask $=00101$, this shows that the bits are wildcard bits on the bit- 0 and bit-2 position of the rule. If the current DBbits $=10100$, this demonstrates that bit- 2 and bit- 4 positions are the distinguishing bits. Thus, we need to omit this bit-2 position since this bit position is shown in the mask field. Consequently, we utilize the bitwise XOR operation to omit the affected bit position. $00101 \wedge 10100=10001$. We note that the bit- 2 of DBbits becomes 0 after the XOR operation, which omits the wildcard bit-2. Then, we employ the extract instruction_pext_u64( ) to extract the bit position value where the bit position is " 1 " in DBbits. For doing so, we obtain the value of extractMask, which represents the affected bit positions excluding the wildcard bits.

Next, we process the searchForInsert function, checking if the new rule is already inserted in the trie data structure to avoid duplicated insertion. First, we traverse the trie from the root node to the leaf node and find a match during this process. For doing the traversal in each level, we extract the partial rule from the inserting rule by utilizing the extract instruction_pext_u64 (rule.value, DBbits) to obtain extractedRst, where DBbits is the discriminative bit set of the current node. If this rule's mask value is 0 , then the partial rule is equal to extractedRst. Otherwise, the partial rule is calculated by _pext_u64(extractedRst, extractMask). By doing this, we omit the wildcard bits to guarantee the correct matching result. If we interpreted the wildcard bit $*$ to either bit- 0 or bit-1, we would lose some of the rules' information. The full procedure of calculating partial rule/key of the whole rule/key is shown in function extractMaskFromsuccessiveByte( ) in Algorithm 1.

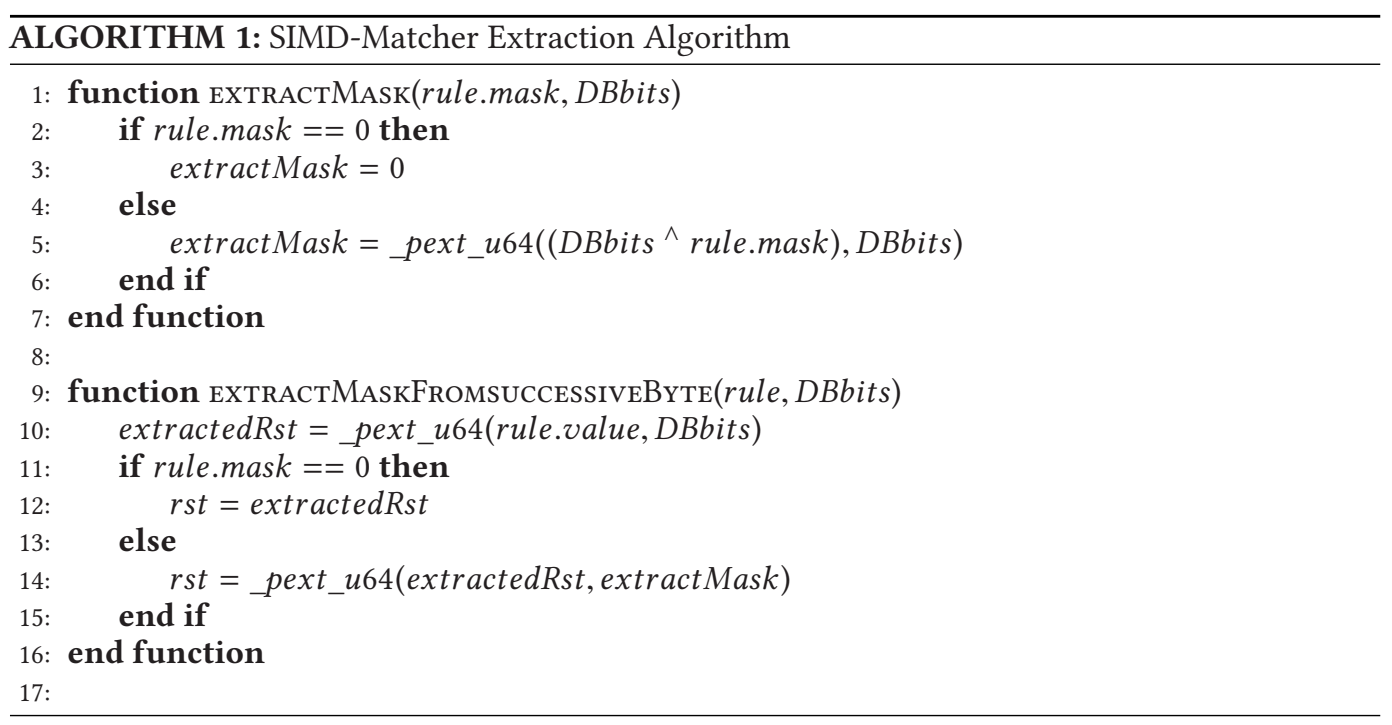


The result of function searchForInsert (root, rule) is the matched rule. Since we only insert a portion of the rule information, we need to compare the matched rule with the inserting rule by utilizing function executeForDiffingKeys( ) to obtain the new missing binary node and update the corresponding node's DBbits. In the original executeForDiffingKeys( ) function, HOT only compares the exact rules/keys. However, for implementing arbitrary matching during the comparisons, we need to consider the wildcard bits. Thus, the new comparison function new_executeForDiffingKeys( ) involves two new arguments: mask field of matched rule and inserting rule, respectively, defined in Definition 3.1.

Definition 3.1. The mismatched binary bit between matched rule oldRule and inserting rule newRule is collected from their wildcard distribution, as defined in Equation (1):

$$
\begin{aligned}
& A=\text { oldRule.value } \mid \text { oldRule.mask } \mid \text { newRule.mask } \\
& B=\text { newRule.value } \mid \text { oldRule.mask } \mid \text { newRule.mask } \\
& \text { flag_mask }= \begin{cases}0, & \text { if } A \neq B \\
1, & \text { if } A=B \& \& \text { newRule.mask }>0,\end{cases}
\end{aligned}
$$

where $A$ and $B$ are defined as the bitwise OR operation between oldRule and newRule. The bitwise OR operation ignores all the wildcard bits from these two different rules, such that we obtain the exact discriminative bit after comparisons. The flag_mask is the identification flag for selecting different insertion procedures. If $A$ is not equal to $B$ and an exact discriminative bit distinguishing between oldRule and newRule, the flag is set to be false. If $A$ equals $B$ and at the same time newRule has wildcard bits, this shows that a new wildcard rule needs to be inserted. In this case, the flag would be set to true. Alternatively, if the flag becomes false, we utilize HOT's insertion procedure. Otherwise, the wildcard rules will be inserted utilizing our proposed procedure. We demonstrate the details later.

First, the rule is a wildcard rule containing wildcard bits. Because we do not insert the wildcard bit position into the trie, we need to insert this wildcard rule into the affected leaf binary nodes. Therefore, we create a fixed-size array for the binary node structure to store wildcard rules as a linked list. Second, we find the affected subtree, which is represented as the first index of the subtree and the subtree's number of entries. We insert the wildcard rule into these affected leaf entries.

Take the ruleset in Table 2 for example. Here we assume $k=32$, the LSB is the bit- 0 position, and BiNode is a binary node. The insertion procedure is shown in Figure 1. For the first rule $R 1: 00101$, we insert it into the root node in Figure 1(a). For the second rule $R 2: 10110$, we find a new discriminative bit position bit-4, and thus two respective BiNode are created. At the same time, the bit position bit-4 is put into DBbits of the root node. Also, $R 1$ and $R 2$ are stored in the leaf BiNode entries, as shown in Figure 1(b). For rule $R 3: 11110$, we extract the bit positions of DBbits of the root node, that is, the bit-4 position of rule $R 3$, and obtain the matched rule $R 2$. Next, we compare the matched rule $R 2$ with rule $R 3$, which produces a new discriminative bit of the bit- 3 position. After inserting rule $R 3$, the trie data structure is shown in Figure $1(\mathrm{c})$. For rule $R 4: 11101$, we extract the bits of DBbits: bit-4 and bit-3 and obtain the matched rule $R 3$. Then we compare the matched rule $R 3$ with rule $R 4$, which creates a new discriminative bit-1 position, as shown in Figure 1(d). Note that DBbits has $\{$ bit-4, bit-3, bit-1\} so far.

For rule $R 5: 0 * 11 *$, we extract DBbits : $\{$ bit-4, bit-3, bit-1\} and obtain $0 * 1$. By traversing the trie root, we obtain the matched rule $R 1$. Next, we compare rule $R 1$ with rule $R 5$ by implementing the new_executeForDiffingKeys( ) function and generate discriminative bit position bit-1. Because the wildcard bit $*$ of rule $R 5$ is at the bit-3 position, flag_mask is false such that this wildcard rule will be inserted using the original insertion procedure. For rule $R 6: 1 * 10 *$, we extract $\{$ bit- 4 , 


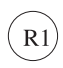

(a)

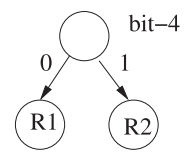

(b)

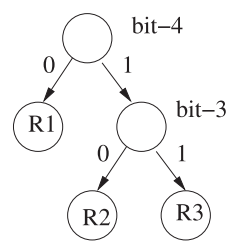

(c)

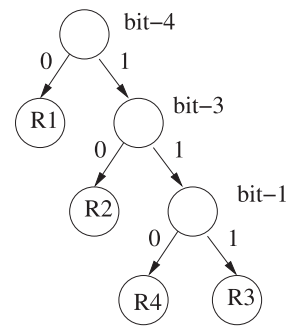

(d)

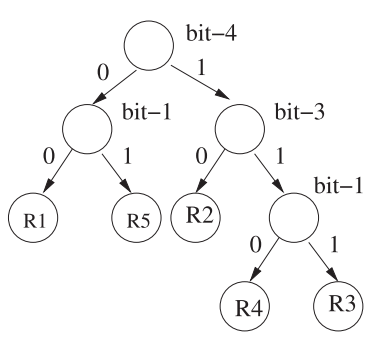

(e)
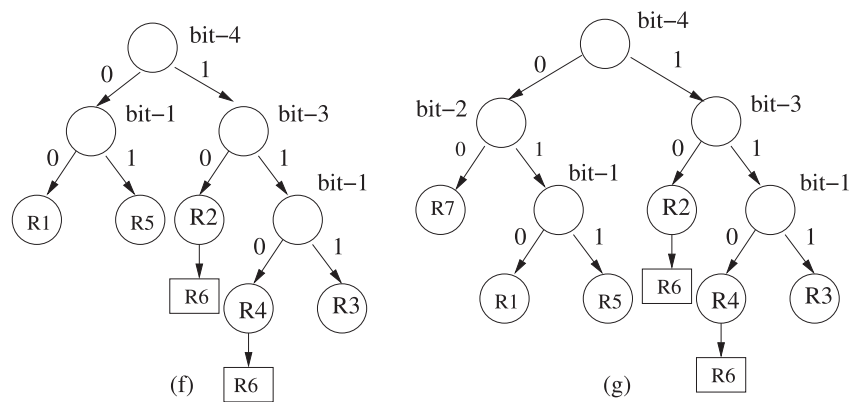

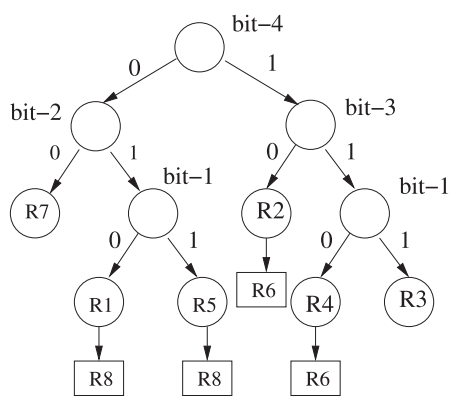

(h)

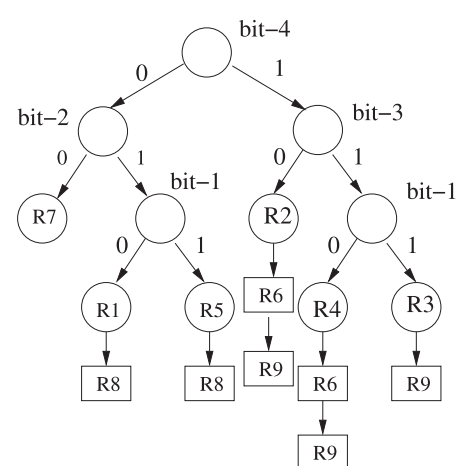

(i)

Fig. 1. Insertion procedure of a wildcard ruleset.

bit-3, bit-1 $\}$ and obtain $1 * 0$, which matches with rule $R 4$ that has the same bits at bit positions $\{$ bit-4, bit-1\}. For comparison between rule $R 4$ and rule $R 6$, oldRule is rule $R 4$, newRule is rule $R 6$, and $A=11101|00000| 01001=11101, B=10100|00000| 01001=11101$. We note that $A=B$; thus, flag_mask is true, which triggers our new insertion procedure. First, we need to find the affected subtree, where the wildcard rule $R 6$ will be inserted. From comparing rule $R 4$ with rule $R 6$, in Figure $1(\mathrm{e})$ we see that rule $R 6$ affects the trie starting from the bit-3 position of rule $R 4$ 's path. Because the bit-3 on rule $R 6$ is a *, it affects the two children " 0 " and " 1 ." There is only one child on the " 0 " path. The leaf entry rule $R 2$ is affected. There are two leaf entries on the " 1 " path: rule $R 4$ and rule $R 3$. Since the bit-1 position of rule $R 6$ is 0 , only rule $R 4$ is affected. Therefore, the number of affected leaf entries is 2: rule $R 2$ and rule $R 4$.

First, we find the affected subtree, whose first entry index of the subtree is 2 , rule $R 2$, counting from the leftmost side and index starting from 0 . The number of affected entries is 2 ; that is, the 
Table 3. An Example of a Prefix Ruleset

\begin{tabular}{|c|c|c|c|}
\hline Rules & Value & Mask & Priority \\
\hline R1: 01001 & 01001 & 00000 & 1 \\
\hline R2: 11100 & 11100 & 00000 & 2 \\
\hline R3: 11110 & 11110 & 00000 & 3 \\
\hline R4: 11011 & 11011 & 00000 & 4 \\
\hline R5: $011^{* *}$ & 01100 & 00011 & 5 \\
\hline R6: $110^{* *}$ & 11000 & 00011 & 6 \\
\hline R7: $00^{* * *}$ & 00000 & 00111 & 7 \\
\hline R8: $01^{* * *}$ & 01000 & 00111 & 8 \\
\hline R9: $1^{* * *}$ & 10000 & 01111 & 9 \\
\hline
\end{tabular}

affected leaf entries are rule $R 2$ and rule $R 4$. Second, we insert this wildcard rule $R 6$ into the leaf entry rule $R 2$ and rule $R 4$. As shown in Figure $1(\mathrm{f})$, the wildcard rule is represented as a rectangle.

For rule $R 7: 0 * 0 * *$, since its bit positions bit-3, bit-1 are all *, we only extract bit-4 and obtain matched rule $R 1$. We compare rule $R 1$ with $R 7$ and produce a new discriminative bit position bit-2, which will be added into the root node's DBbits. Thus, DBbits has $\{$ bit-4, bit-3, bit-2, bit-1 $\}$. The current trie is shown in Figure $1(\mathrm{~g})$. For rule $R 8: 0 * 1 * *$, we extract the non-wildcard bit position: bit-4 and bit-2, and obtain the matched rule $R 1$. We compare $R 1$ with $R 8$ by utilizing Equation (1), $A=00101|00000| 01011=01111, B=00100|00000| 01011=01111$. We note that $A=B$; thus, flag_mask is true, which triggers our new insertion procedure. For rule $R 8$, the affected subtree mask is 000110 , which shows that the affected leaf entries are index 1 and index 2, which are rule $R 1$ and rule $R 5$, as shown in Figure $1(\mathrm{~g})$.

For the last rule $R 9: 1 * * * *$, we extract the non-wildcard bit position: bit-4, and obtain matched rule $R 4$. We compare rule $R 6$ with $R 9$ and calculate flag_mask, whose value is 1 . Next, we collect the affected subtree. Since rule $R 9$ has wildcards $*$ on all bit positions except bit-4, we note that the affected leaf entries are rule $R 2$, rule $R 4$, and rule $R 3$. Thus, we insert this new wildcard rule $R 9$ into the wildcard rule list under these three leaf entries. The final trie is shown in Figure 1(i). Note that we insert seven wildcard rules into the wildcard rule list container of the affected leaf entries, which guarantees that all of the rule information is inserted into the trie data structure.

For the ruleset in Table 2, we see that the wildcard bits $*$ are at random positions and not always at the end. Since the trie is a lexicographic data structure, different positions of wildcard bits build various data structures. This is because the rules' bits are inserted in order from the MSB to LSB. If the wildcard bit is on the upper level, this will affect more leaf entries, resulting in more insertion of wildcard rules. If the ruleset is changed to all prefix rules, the number of inserted wildcard rules will be decreased. The ruleset in Table 3 is a transform of the ruleset in Table 2. Its insertion procedure is shown in Figure 2.

Comparing the final trie between Figure 2(i) and Figure 1(i), we observe that the two trie data structures are different. First, the DBbits of each rule are different. We take rule $R 4$ as an example. In Figure 1(i) rule $R 4$ is composed of $\{$ bit-4, bit-3, bit-1\}, while in Figure 2(i) rule $R 4$ consists of $\{$ bit-4, bit-2\}. Second, in Figure 1(i) there are seven wildcard rules inserted into the trie, whereas in Figure 2(i) only six wildcard rules are inserted. This is because Figure 2(i) has all prefix rules, but Figure 1(i) has arbitrary rules that have wildcard bits $*$ at any position. However, the height of the trie is the same. Thus, in contrast to arbitrary rules, prefix rules consume less memory.

\subsection{Search Operation}

For a search operation, we traverse from the root node to the leaf node of the trie. The procedure is as follows: 


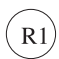

(a)

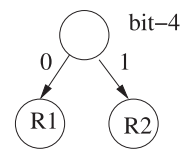

(b)

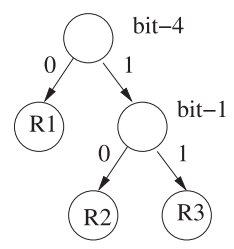

(c)

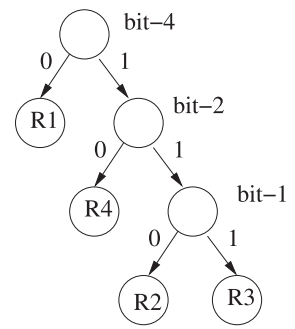

(d)

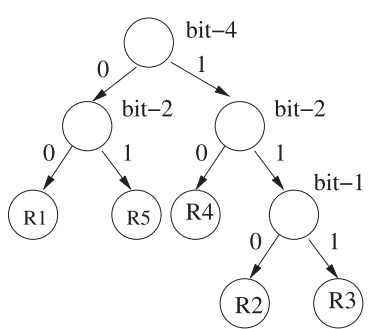

(e)

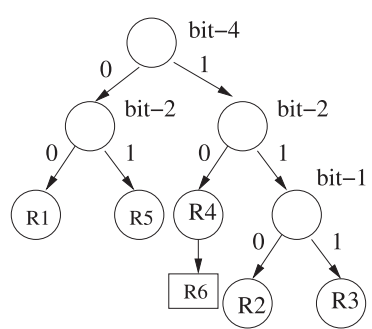

(f)

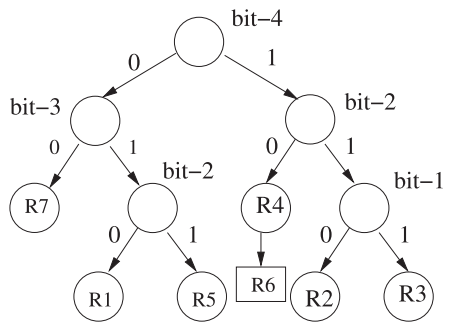

(g)

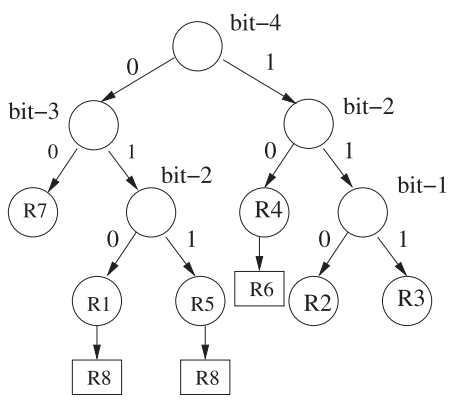

(h)

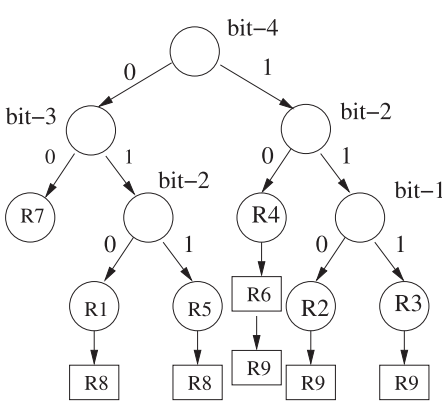

(i)

Fig. 2. Insertion procedure of a prefix ruleset.

(1) We extract the partial key from the incoming key by utilizing the search node's discriminative bits set.

(2) We utilize SIMD comparison instructions to compare the extracted partial key with the trie node's entries. The Pseudocode of SIMD-based search operation is represented in Algorithm 2.9

(3) After the comparison, if we find the matched item, we will move to the next level and go back to step (2), and we will not go to step (4) until the leaf node is reached. Otherwise, it is a non-match, return false.

(4) We compare the matched entry with the incoming key in terms of the full bits to check if there is an accurate matching. If it is a matching, then return true. Otherwise, check if the size of the wildcardRuleList is empty. If it is empty, then return false. Otherwise, the

${ }^{9}$ We extended the algorithm and code proposed by Binna et al. [5]. 


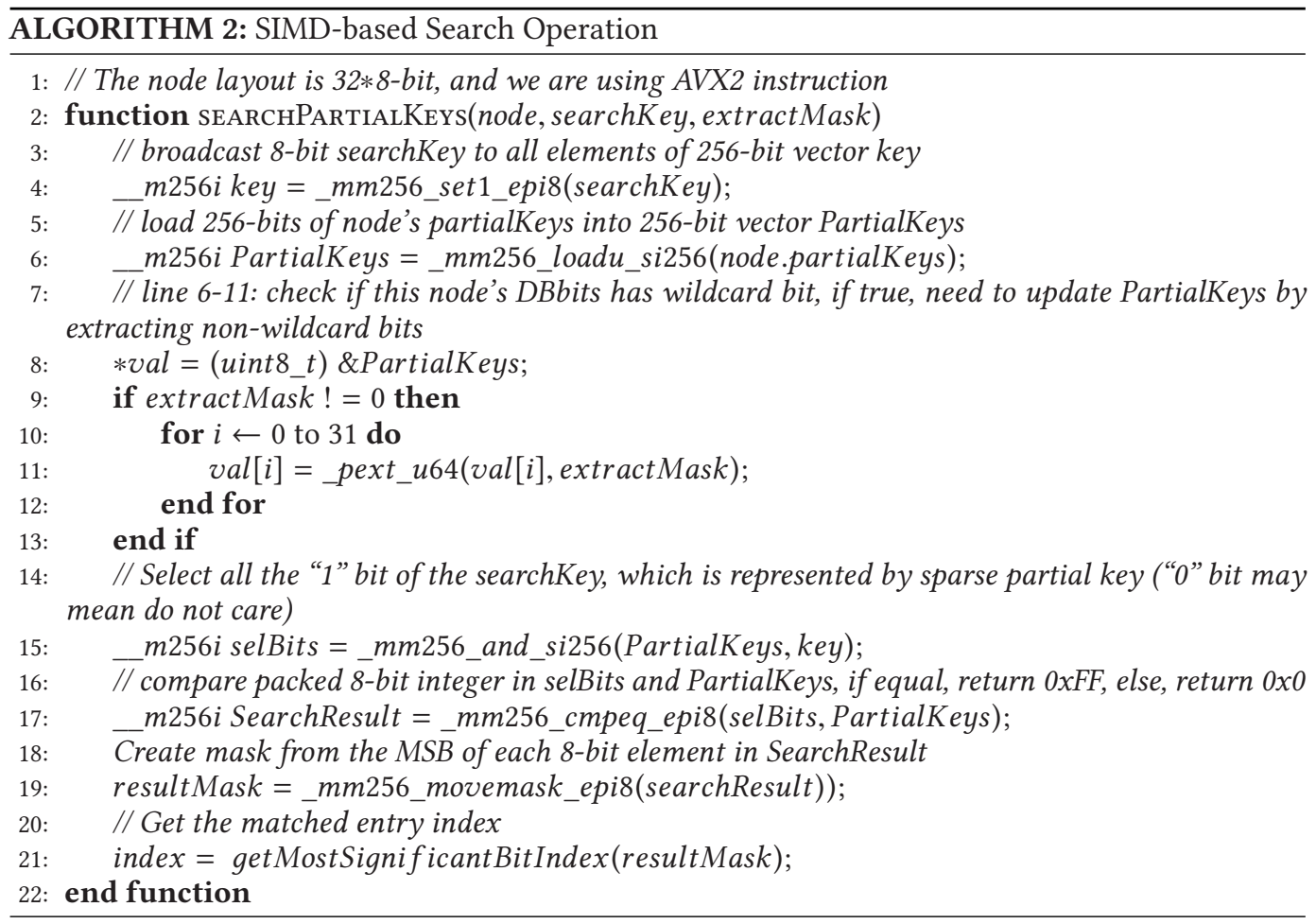

wildcard rules in the list need to be compared with the incoming key. The matching item will be the final result.

Our proposed SIMD-Matcher extracts the effective discriminative bits to guarantee accurate partial rule insertion to support wildcard matching in HOT. During searching, SIMD-Matcher can obtain the correct match result due to the wildcard rule array.

\section{EVALUATION}

This section evaluates SIMD-Matcher on rulesets generated from packet capture (PCAP) traces and random generator. SIMD-Matcher is our advanced arbitrary matching framework, which is SIMD and cache-friendly. We first present the evaluation methodology, followed by performance analysis. Finally, we deliver the scalability of the SIMD-Matcher.

\subsection{Methodology}

We design SIMD-Matcher in C++ and employ two different rule generation methods to generate the rulesets. The rules are 64-bit integers generated from the IPv4 source and destination addresses; nonetheless, our solution can also be extended to support IPv6 after proper adjustment. ${ }^{10}$ First, we employ a heuristic rule generation method [22] to synthesize the PCAP rulesets, where 11,000 rules are generated based on the CAIDA PCAP data [7]. We choose five various set samples with

\footnotetext{
${ }^{10}$ The CAIDA traces in our presented experiments use the IPv4 protocol. However, our approach can flexibly support IPv6 after adopting these changes: Since IPv6 addresses comprise more bits (128 bits), we need to scale up the data structure. The array size of the trie node is determined by the number of distinguished bits between all rules represented by 128 bits If the number of distinguished bits is smaller than 64 , then the $k$ can be set to 64 instead of 32 . We can keep the same node layout, with an increased array size of 64 .
} 
Table 4. Evaluation Parameters

\begin{tabular}{|c|c|}
\hline small_keySet & 10,000 \\
\hline medium_keySet & $100,000,000$ \\
\hline large_keySet & $1000,000,000$ \\
\hline small_ruleSet & 10,000 \\
\hline large_ruleSet & 100,000 \\
\hline
\end{tabular}

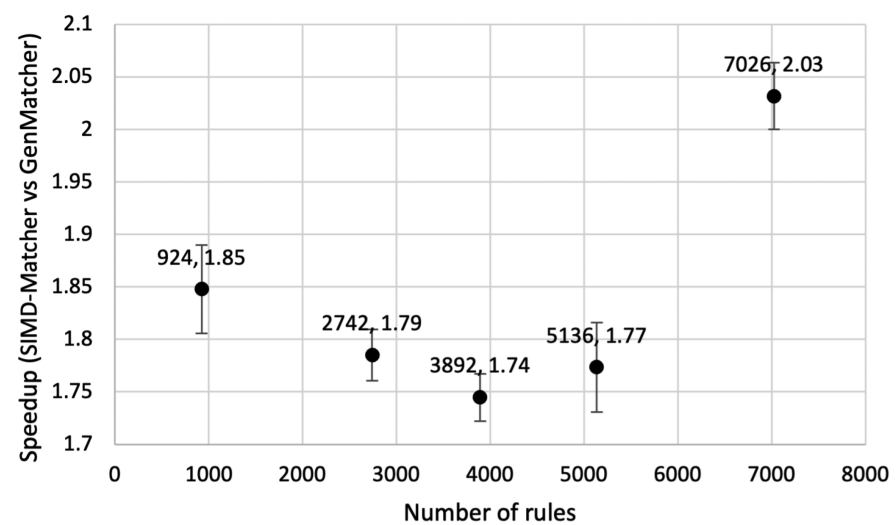

Fig. 3. Search time performance comparison with respect to different number of rules on a small scale.

different sizes (924, 2,742, 3,892, 5,136, 7,062) and extract a unique set of 1,044,618 keys from the traces to show the scalable performance.

Second, we use our random rule generator to create two different rule table sizes $(10,000,100,000)$ and four different key set sizes $(10,000,100,000,100,000,000,1000,000,000)$ with various match ratios: $\{0.5 \%, 1 \%, 2.5 \%, 5 \%, 10 \%, 25 \%, 50 \%\}$, that is, the percentage of keys that find a match. With $30 \%$ wildcard rules, the number of wildcard rules is calculated as $30 \% \times$ rule size. Speedup is defined as GenMatcher search time/SIMD-Matcher search time. Table 4 shows the Evaluation Parameters. We evaluate SIMD-Matcher on a real system, with an Intel i9-9900 16-core 3.1GHz processor and 32 GB DRAM. The cache hierarchy consists of a 32k L1d, 32k L1i, 256k L2, and 16384k L3 cache. ${ }^{11}$ The presented results take the average of 10 separate simulation runs.

\subsection{Performance Comparisons}

We compare SIMD-Matcher against GenMatcher in terms of search time, insert time, and memory cost.

4.2.1 Search Time. We utilize two different benchmarks to evaluate search time performance. We evaluate the performance by employing the ruleset and key set generated by PCAP traces. The search time performance is represented in Figure 3. This simulation evaluates five different rule sizes $(924,2,742,3,892,5,136,7,062)$. Note that with the increasing number of rules, the speedup of SIMD-Matcher against GenMatcher is not linear because the trie height mainly determines search time. Table 5 shows the trie heights and match ratios of the corresponding test cases. With

\footnotetext{
${ }^{11}$ Note: Our baseline implementation of SIMD-Matcher is single threaded, though multi-threaded implementations are possible; we leave this to future work.
} 
Table 5. Parameters of the Trie

\begin{tabular}{|c|c|c|c|c|c|}
\hline \#Rules & 924 & 2,742 & 3,892 & 5,136 & 7,026 \\
\hline Trie height & 2 & 3 & 3 & 3 & 3 \\
\hline Match ratio & $8 \%$ & $31.1 \%$ & $25.3 \%$ & $29.8 \%$ & $37.8 \%$ \\
\hline
\end{tabular}

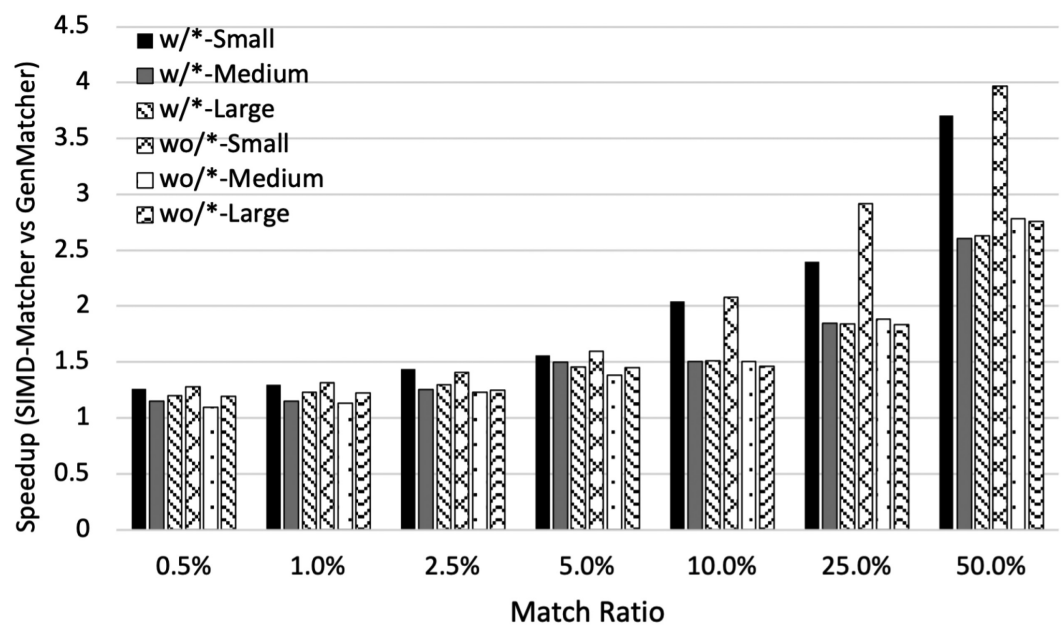

Fig. 4. Search time performance comparison with respect to different number of keys on a large scale.

the same trie height, the larger the match ratio, the more significant the speedup. For the cases $(2,742,3,892,5,136,7,062)$, their trie height is three. Case 7,062 has the largest speedup, of 2.03X, since its match ratio has the most significant value, 37.8\%. We observe that case 924 has a trie height of two, which is smaller than other cases' height of three. While we expected this to lead it to outperform the other cases, it did not beat case 7,026. This is because the match ratio of case 924 is only $8 \%$, and thus it requires more comparisons.

We evaluate the search time performance by utilizing the randomly generated, 64-bit rule benchmark on a larger scale. Figure 4 shows the search time speedup with respect to SIMD-Matcher and GenMatcher. The number of rules is 10,000 , the size of small_keySet is 10,000, the size of medium_keySet is 100,000,000 and the size of large_keyset is 1,000,000,000.

First, in the figure, we see that SIMD-Matcher produces substantially better results than GenMatcher on both with $30 \%$ wildcard rule and without wildcard rule scenarios. This is because the search time performance of both SIMD-Matcher and GenMatcher is dominated by the search depth of their data structure, while the average search depth of SIMD-Matcher is smaller than GenMatcher.

Second, we observe that SIMD-Matcher provides greater speedups for larger match ratios. In the SIMD-Matcher framework, we add a fixed-size array in the leaf node entries. This array stores the wildcard rules. During a search process, the key needs to traverse the trie from the root node to the leaf node. If the matched leaf node entry has a non-empty array, the key needs to search through the array sequentially. If the match result is a non-match, the key searches through the whole array. Otherwise, the key might only search a portion of the array. Therefore, a non-match consumes more comparisons than a matching, and thus the larger the match ratio, the greater the speedup. 


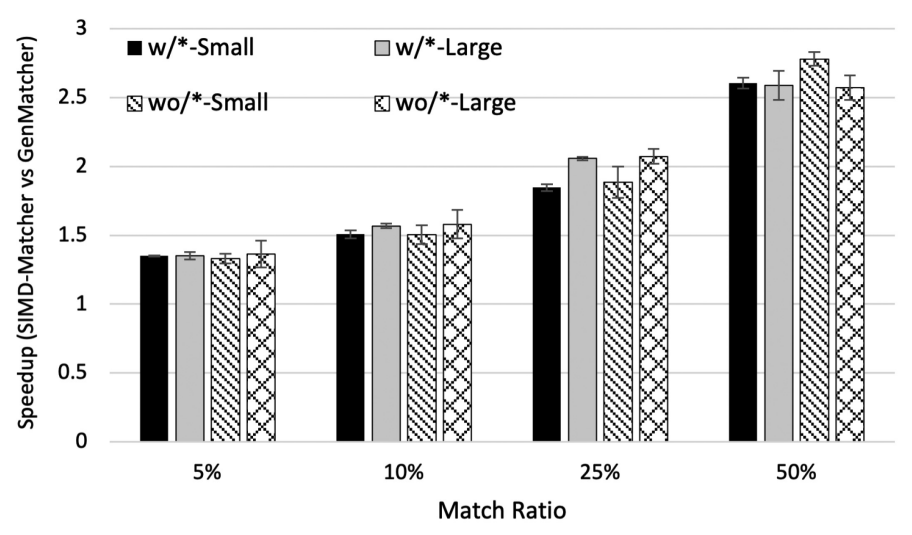

Fig. 5. Search time performance comparison with respect to different number of rules.

Table 6. Number of Wildcard Entries Inserted in the Trie

\begin{tabular}{|c|c|c|c|c|c|}
\hline \#Rules & 924 & 2,742 & 3,892 & 5,136 & 7,026 \\
\hline \# Wild_rule entries & 2 & 1,235 & 837 & 1,989 & 3,783 \\
\hline
\end{tabular}

Third, Figure 4 shows that the benchmark without wildcard rules has a slightly better performance than the benchmark with $30 \%$ wildcard rules when the match ratio is greater than or equal to $5 \%$. This is because no array is inserted in the leaf node entries, which accelerates the search process and results in a greater speedup.

Last, we see that under a fixed-size ruleset, the speedup with small_keySet is much more significant than medium_keySet and large_keySet. This is because of the system running overhead. The speedup is achieving stability when the key size becomes sufficiently large enough to minimize the overhead impact. Here we see no big difference between the medium_keySet $(100,000,000)$ and the large_keySet $(1000,000,000)$. The search time speedup is up to $2.7 \mathrm{X}$.

Figure 5 shows the search time speedup concerning two different rule sizes. The number of keys is $100,000,000$, the size of small_ruleSet is 10,000 , and the size of large_ruleSet is 100,000 . As shown in Figure 5, we see that the speedup performance of large_ruleSet is greater than small_ruleSet on match ratio $\{5 \%, 10 \%, 25 \%\}$. However, in the $50 \%$ case, the large_ruleSet speedup is smaller than the small_ruleSet. Since the GenMatcher data structure is a binary trie, each rule's depth is equal to its non-wildcard bits. The maximal depth for a rule is 64 because the data is 64 -bit integers. The depth of the SIMD-Matcher data structure is determined by the relationship between the rules since we build the data structure using their discriminative bits. Therefore, there is no linear trend toward the increasing number of rules. This shows the scalability of our SIMD-Matcher.

4.2.2 Insert Time. We evaluate the insert time performance utilizing two sets of benchmarks. For the first set, we employ the ruleset and key generated by PCAP traces, as shown in Figure 6(a). For the other set, we utilize the ruleset and key set created by a random generator as shown in Figure 6(b). Figure 6(a) shows the speedup comparing SIMD-Matcher against GenMatcher. The insert time is determined by the number of rules and the inserted number of wildcard entries. In Figure 6(a), note that the 924 rule case has the best speedup since the rule size is the smallest and the number of wildcard entries is only two. Table 6 shows the number of wildcard entries inserted in the trie. 


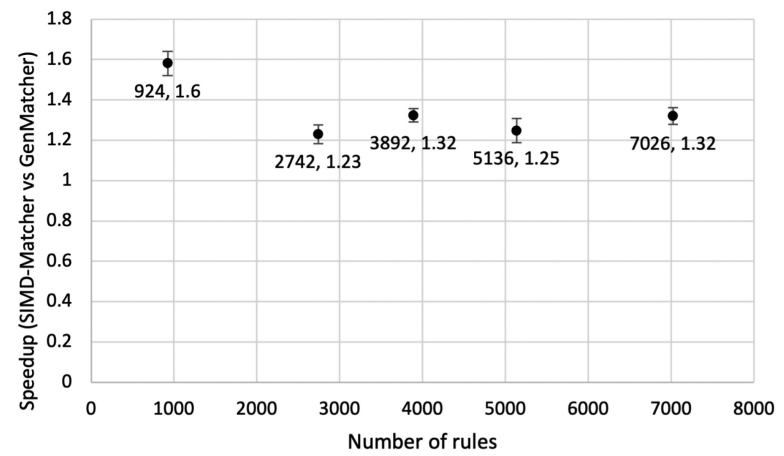

(a) Rule size under $10 \mathrm{k}$

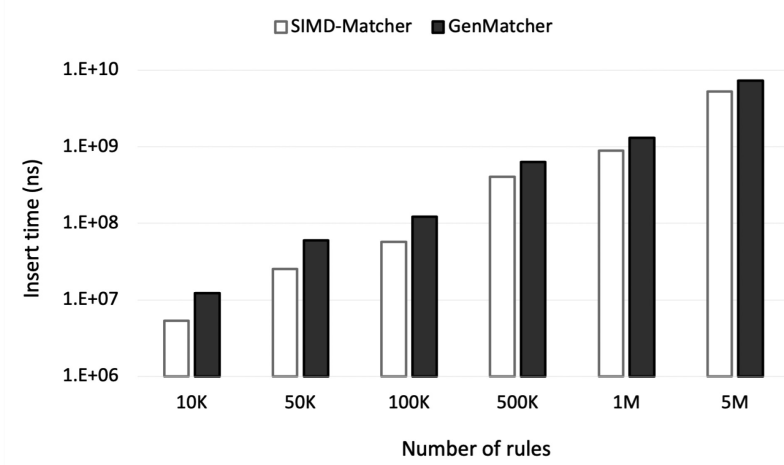

(b) Rule size over $10 \mathrm{k}$

Fig. 6. Insert time performance comparison.

Table 7. Insert Time Speedup between SIMD-Matcher and GenMatcher on a Large Scale

\begin{tabular}{|c|c|c|c|c|c|c|}
\hline \#Rules & $10 \mathrm{~K}$ & $50 \mathrm{~K}$ & $100 \mathrm{~K}$ & $500 \mathrm{~K}$ & $1 \mathrm{M}$ & $5 \mathrm{M}$ \\
\hline Speedup & 2.31 & 2.37 & 2.14 & 1.56 & 1.48 & 1.38 \\
\hline
\end{tabular}

Figure 6(b) represents the insert time performance on a larger scale. Table 7 shows the insert time speedup of SIMD-Matcher versus GenMatcher in the large scale. As shown in Table 7, the speedup is decreasing with the increasing number of rules. Nonetheless, inserting 5,000,000 rules is about 7.3 seconds, and the speedup is about 1.38 . Thus, the insert time performance of a large-scale dataset is stable.

4.2.3 Memory Cost. Figure 7(a) shows the memory cost for five different rule sets on a small scale. In the figure, we note that the memory cost increases with the increasing number of rules. For GenMatcher, we insert all the non-wildcard bits into the trie. In contrast to GenMatcher, SIMDMatcher only inserts the discriminative bits into the trie. Therefore, in general, SIMD-Matcher saves more memory than GenMatcher. Also, SIMD-Matcher needs to insert some wildcard entries when there are no discriminative bits between the rules with wildcard bits. 


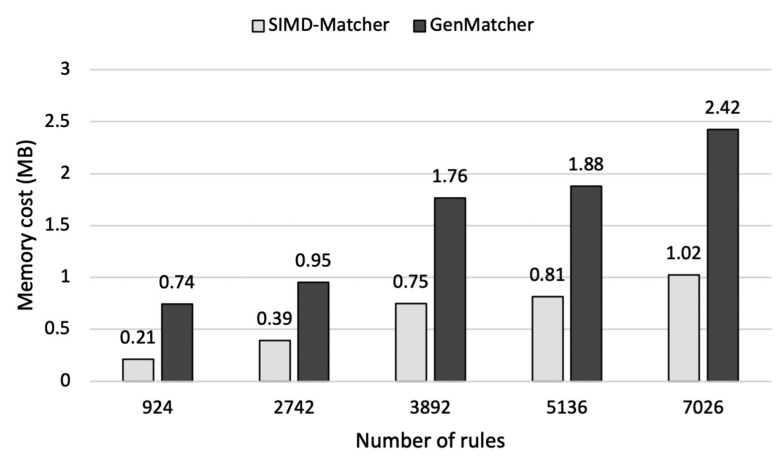

(a) Rule size under $10 \mathrm{k}$

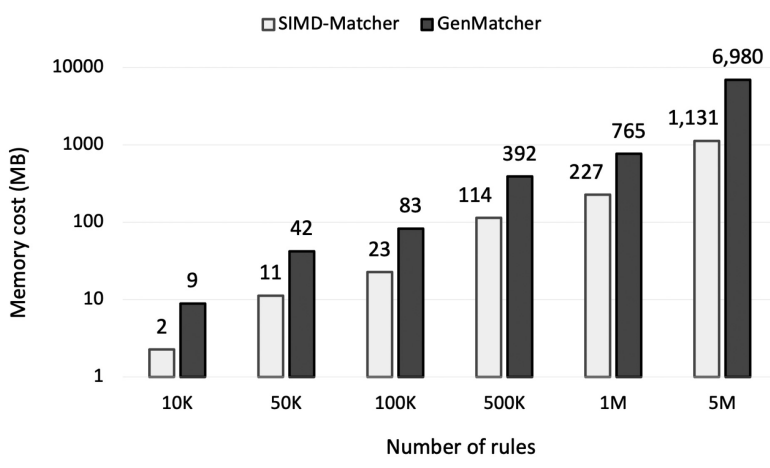

(b) Rule size over $10 \mathrm{k}$

Fig. 7. Memory cost comparison.

Table 8. Memory Cost Comparisons between SIMD-Matcher and GenMatcher

\begin{tabular}{|c|c|c|c|c|c|c|c|c|c|c|c|}
\hline \#Rules & 924 & 2,742 & 3,892 & 5,136 & 7,026 & $10 \mathrm{~K}$ & $50 \mathrm{~K}$ & $100 \mathrm{~K}$ & $500 \mathrm{~K}$ & $1 \mathrm{M}$ & $5 \mathrm{M}$ \\
\hline Reduction & 3.53 & 2.44 & 2.36 & 2.31 & 2.37 & 3.94 & 3.74 & 3.65 & 3.45 & 3.37 & 6.17 \\
\hline
\end{tabular}

Figure 7(b) shows the memory cost performance with respect to SIMD-Matcher and GenMatcher on a large scale up to 5,000,000 rules. In the figure, the memory cost is represented in log scale. From the figure we see that the trend is consistent with Figure 7(a). Table 8 shows the reduction of memory cost with respect to SIMD-Matcher and GenMatcher. Note that the reduction becomes larger with the increasing number of rules. The reduction is up to $6.17 \mathrm{X}$ when the number of rules is $5,000,000$.

\subsection{Scalability}

In the evaluation, we utilize two different sets of benchmarks to cover different scales of rule sets and key sets. We evaluate the performance by scaling the rule size from 924 up to 5 million rules for the ruleset. For the key set, we demonstrate the performance by scaling from 10,000 up to $1,000,000,000$ search operations. With respect to search time performance, compared to GenMatcher, SIMD-Matcher achieves up to 2.7X when the rule size is 100,000 and key size is 
1,000,000,000. For insert time performance, SIMD-Matcher outperforms GenMatcher because of the partial rules insertion and SIMD-based comparisons. The insert time is about 7.3 seconds when the rule size is 5,000,000. For memory cost, SIMD-Matcher consumes less memory than GenMatcher. For a rule size of 5,000,000, the memory cost is about $1.11 \mathrm{~GB}$.

\section{CONCLUSIONS}

This article proposes SIMD-Matcher, an efficient SIMD and cache-friendly arbitrary matching mechanism. SIMD-Matcher interprets arbitrary rules into three fields, value, mask, and priority, to be able to insert into an advanced trie-based data structure. SIMD-Matcher employs our proposed extraction algorithm to process the wildcard bits and insert arbitrary rules with randomly positioned wildcards. To guarantee accurate match results with wildcard rules, SIMD-Matcher adds an array of wildcard entries to the leaf entries, which stores the wildcard rules. Experiments show that SIMD-Matcher achieves search time speedup by utilizing the SIMD feature on average by $2.7 \mathrm{X}$ compared to GenMatcher, and up to 6.17X reduction for the memory footprint.

\section{REFERENCES}

[1] 2008. Intel AVX: New frontiers in performance improvements and energy efficiency. While Paper (2008). Computer Engineering and Information Technology Department, Razi University, Kermanshah, Iran.

[2] Hamed Alimohammadi and Mahmood Ahmadi. 2020. Common non-wildcard portion-based partitioning approach to SDN many-field packet classification. Computer Networks 181 (2020), 107534. https://doi.org/10.1016/j.comnet.2020. 107534

[3] Nikolas Askitis and Ranjan Sinha. 2007. HAT-trie: A cache-conscious trie-based data structure for strings. In Proceedings of the 30th Australasian Conference on Computer Science - Volume 62 (ACSC'07). Australian Computer Society, Inc., AUS, 97-105.

[4] Ramazan S. Aygün. 2008. S2S: Structural-to-syntactic matching similar documents. Knowledge and Information Systems 16, 3 (Sept. 2008), 303-329.

[5] Robert Binna, Eva Zangerle, Martin Pichl, Günther Specht, and Viktor Leis. 2018. HOT: A height optimized trie index for main-memory database systems. In Proceedings of the 2018 International Conference on Management of Data (SIGMOD'18). Association for Computing Machinery, New York, NY, 521-534. https://doi.org/10.1145/3183713.3196896

[6] Richard Cole, Lee-Ad Gottlieb, and Moshe Lewenstein. 2004. Dictionary matching and indexing with errors and don't cares. In Proceedings of the 36th Annual ACM Symposium on Theory of Computing (STOC'04). Association for Computing Machinery, New York, NY, 91-100. https://doi.org/10.1145/1007352.1007374

[7] Paul Hick and Dan Andersen. 2012. The CAIDA Anonymized 2012 Internet Traces - 2012. http://www.caida.org/data/ passive/passive_2012_dataset.xml.

[8] Susumu Date, Hirotake Abe, Dashdavaa Khureltulga, Keichi Takahashi, Yoshiyuki Kido, Yasuhiro Watashiba, Pongsakorn U-Chupala, Kohei Ichikawa, Hiroaki Yamanaka, Eiji Kawai, and Shinji Shimojo. 2015. An empirical study of SDN-accelerated HPC infrastructure for scientific research. In Proceedings of the 2015 International Conference on Cloud Computing Research and Innovation (ICCCRI'15). IEEE Computer Society, 89-96. https://doi.org/10.1109/ICCCRI. 2015.13

[9] EventHelix. 2017. Why software developers should care about CPU caches. https://medium.com/software-design/ why-software-developers-should-care-about-cpu-caches-8da04355bb8a.xml.

[10] A. Fiessler, S. Hager, and B. Scheuermann. 2017. Flexible line speed network packet classification using hybrid on-chip matching circuits. In 2017 IEEE 18th International Conference on High Performance Switching and Routing (HPSR'17). IEEE, 1-8. https://doi.org/10.1109/HPSR.2017.7968678

[11] Open Networking Foundation. 2015. OpenFlow Switch Specification. Technical Report. ONF.

[12] Matteo Frigo, Charles E. Leiserson, Harald Prokop, and Sridhar Ramachandran. 1999. Cache-oblivious algorithms. In Proceedings of the 40th Annual Symposium on Foundations of Computer Science (FOCS'99). IEEE Computer Society, 285.

[13] P. Goransson and C. Black. 2014. Software defined networks: A comprehensive approach. In Software Defined Networks: A Comprehensive Approach, 1-325.

[14] C. L. Hsieh and N. Weng. 2016. Many-field packet classification for software-defined networking switches. In 2016 ACM/IEEE Symposium on Architectures for Networking and Communications Systems (ANCS'16). IEEE, 13-24. https: //doi.org/10.1145/2881025.2881036

[15] Changkyu Kim, Jatin Chhugani, Nadathur Satish, Eric Sedlar, Anthony D. Nguyen, Tim Kaldewey, Victor W. Lee, Scott A. Brandt, and Pradeep Dubey. 2010. FAST: Fast architecture sensitive tree search on modern CPUs and GPUs. 
In Proceedings of the 2010 ACM SIGMOD International Conference on Management of Data (SIGMOD'10). Association for Computing Machinery, New York, NY, 339-350. https://doi.org/10.1145/1807167.1807206

[16] Thomas Kissinger, Benjamin Schlegel, Dirk Habich, and Wolfgang Lehner. 2012. KISS-tree: Smart Latch-free inmemory indexing on modern architectures. In Proceedings of the 8th International Workshop on Data Management on New Hardware (DaMoN'12). Association for Computing Machinery, New York, NY, 16-23. https://doi.org/10.1145/ 2236584.2236587

[17] S. V. Krishna, A. Shrivastava, and S. J. Wagh. 2017. SDN in high performance computing for scientific and business environment (SBE). In 2017 International Conference on Computational Intelligence in Data Science (ICCIDS'17). IEEE, 1-8. https://doi.org/10.1109/ICCIDS.2017.8272654

[18] Kuan-Tsen Kuo, Charles H.-P. Wen, Cheng Suo, and I-Chen Tsai. 2015. SWF: Segmented wildcard forwarding for flow migration in OpenFlow datacenter networks. In 2015 IEEE International Conference on Communications (ICC'15). 313-318. https://doi.org/10.1109/ICC.2015.7248340

[19] V. Leis, A. Kemper, and T. Neumann. 2013. The adaptive radix tree: ARTful indexing for main-memory databases. In 2013 IEEE 29th International Conference on Data Engineering (ICDE'13). IEEE, 38-49. https://doi.org/10.1109/ICDE 2013.6544812

[20] Yadi Ma and Suman Banerjee. 2012. A smart pre-classifier to reduce power consumption of TCAMs for multidimensional packet classification. SIGCOMM Computer Communication Review 42, 4 (Aug. 2012), 335-346. https: //doi.org/10.1145/2377677.2377749

[21] Markus Mäsker, Tim Süß, Lars Nagel, Lingfang Zeng, and André Brinkmann. 2019. Hyperion: Building the largest in-memory search tree. In Proceedings of the 2019 International Conference on Management of Data (SIGMOD'19). Association for Computing Machinery, New York, NY, 1207-1222. https://doi.org/10.1145/3299869.3319870

[22] Luke Mchale, Jasson Case, Paul V. Gratz, and Alex Sprintson. 2014. Stochastic pre-classification for SDN data plane matching. In Proceedings of the 2014 IEEE 22nd International Conference on Network Protocols (ICNP'14). IEEE Computer Society, 596-602. https://doi.org/10.1109/ICNP.2014.95

[23] C. R. Meiners, A. X. Liu, and E. Torng. 2012. Bit weaving: A non-prefix approach to compressing packet classifiers in TCAMs. IEEE/ACM Transactions on Networking 20, 2 (April 2012), 488-500. https://doi.org/10.1109/TNET.2011.2165323

[24] Donald R. Morrison. 1968. PATRICIA-Practical algorithm to retrieve information coded in alphanumeric. Fournal of the ACM 15, 4 (Oct. 1968), 514-534. https://doi.org/10.1145/321479.321481

[25] Nadia Pisanti, Maxime Crochemore, Roberto Grossi, and Marie-France Sagot. 2005. Bases of motifs for generating repeated patterns with wild cards. IEEE/ACM Transactions on Computational Biology and Bioinformatics 2, 1 (Jan. 2005), 40-50. https://doi.org/10.1109/TCBB.2005.5

[26] Y. Qu, S. Zhou, and V. K. Prasanna. 2013. Scalable many-field packet classification on multi-core processors. In 2013 25th International Symposium on Computer Architecture and High Performance Computing. 33-40. https://doi.org/10 1109/SBAC-PAD.2013.29

[27] Jun Rao and Kenneth A. Ross. 1999. Cache conscious indexing for decision-support in main memory. In Proceedings of the 25th International Conference on Very Large Data Bases (VLDB'99). Morgan Kaufmann Publishers Inc., 78-89.

[28] M. A. Ruiz-Sanchez, E. W. Biersack, and W. Dabbous. 2001. Survey and taxonomy of IP address lookup algorithms. IEEE Network 15, 2 (March 2001), 8-23.

[29] Tong Shen, Da-Fang Zhang, Gaogang Xie, and Xin-Yi Zhang. 2018. Optimizing multi-dimensional packet classification for multi-core systems. Journal of Computer Science and Technology 33, 5 (2018), 1056-1071. https://doi.org/10.1007/ s11390-018-1873-9

[30] Sajad Shirali-Shahreza and Yashar Ganjali. 2015. ReWiFlow: Restricted wildcard openflow rules. SIGCOMM Computer Communication Review 45, 5 (Sept. 2015), 29-35. https://doi.org/10.1145/2831347.2831352

[31] S. Thakkur and T. Huff. 1999. Internet streaming SIMD extensions. Computer 32, 12 (1999), 26-34. https://doi.org/10. $1109 / 2.809248$

[32] Ping Wang, Luke McHale, Paul V. Gratz, and Alex Sprintson. 2018. GenMatcher: A generic clustering-based arbitrary matching framework. ACM Transactions on Architecture and Code Optimization 15, 4, Article 51 (Nov. 2018), 22 pages. https://doi.org/10.1145/3281663

[33] Fang Yu, R. H. Katz, and T. V. Lakshman. 2004. Gigabit rate packet pattern-matching using TCAM. In Proceedings of the 12th IEEE International Conference on Network Protocols, 2004 (ICNP'04). IEEE, 174-183. https://doi.org/10.1109/ ICNP.2004.1348108

Received August 2021; revised January 2022; accepted January 2022 